\title{
EXTRACTION OF CRITICAL POINTS OF SMOOTH FUNCTIONS ON BANACH SPACES
}

\author{
MIGUEL GARCÍA-BRAVO
}

\begin{abstract}
Let $E$ be an infinite-dimensional separable Hilbert space. We show that for every $C^{1}$ function $f: E \rightarrow \mathbb{R}^{d}$, every open set $U$ with $C_{f}:=\{x \in E: D f(x)$ is not surjective $\} \subset U$ and every continuous function $\varepsilon: E \rightarrow(0, \infty)$ there exists a $C^{1}$ mapping $\varphi: E \rightarrow \mathbb{R}^{d}$ such that $\|f(x)-\varphi(x)\| \leq$ $\varepsilon(x)$ for every $x \in E, f=\varphi$ outside $U$ and $\varphi$ has no critical points $\left(C_{\varphi}=\emptyset\right)$. This result can be generalized to the case where $E=c_{0}$ or $E=l_{p}, 1<p<\infty$. In the case $E=c_{0}$ it is also possible to get that $\|D f(x)-D \varphi(x)\| \leq \varepsilon(x)$ for every $x \in E$.
\end{abstract}

\section{INTRODUCTION AND MAIN RESULTS}

Our goal in this paper is to prove the following result:

Theorem 1.1. Let $E$ be one of the classical infinite-dimensional Banach spaces $c_{0}$ or $l_{p}$ with $1<p<$ $\infty$. Let $f: E \rightarrow \mathbb{R}^{d}$ be a $C^{1}$ function and $\varepsilon: E \rightarrow(0, \infty)$ a continuous function. Take any open set $U$ containing the critical set of points of $f$, that is $C_{f}:=\{x \in E: D f(x)$ is not surjective $\}$. Then there exists a $C^{1}$ function $\varphi: E \rightarrow \mathbb{R}^{d}$ such that,

(1) $\|f(x)-\varphi(x)\| \leq \varepsilon(x)$ for all $x \in E$;

(2) $f(x)=\varphi(x)$ for all $x \in E \backslash U$;

(3) $D \varphi(x)$ is surjective for all $x \in E$, i.e. $\varphi$ has no critical points; and

(4) in the case that $E=c_{0}$ we also have that $\|D f(x)-D \varphi(x)\| \leq \varepsilon(x)$ for all $x \in E$.

We can make $\varphi$ be of class $C^{k}$ inside the open set $U$, where $k$ denotes the order of smoothness of the space $l_{p}, 1<p<\infty$ or $c_{0}$. A brief explanation of this fact can be found in Remark 4.2.

This theorem is a particular case of the following two more technical results.

Theorem 1.2. Let $E$ be an infinite-dimensional Banach space with an unconditional basis and with $a C^{1}$ equivalent norm $\|\cdot\|$ that locally depends on finitely many coordinates. Let $f: E \rightarrow \mathbb{R}^{d}$ be a $C^{1}$ function and $\varepsilon: E \rightarrow(0, \infty)$ a continuous function. Take any open set $U$ such that $C_{f} \subset U$. Then there exists a $C^{1}$ function $\varphi: E \rightarrow \mathbb{R}^{d}$ such that,

(1) $\|f(x)-\varphi(x)\| \leq \varepsilon(x)$ for all $x \in E$;

(2) $f(x)=\varphi(x)$ for all $x \in E \backslash U$;

(3) $\|D f(x)-D \varphi(x)\| \leq \varepsilon(x)$ for all $x \in E$; and

(4) $D \varphi(x)$ is surjective for all $x \in E$.

Theorem 1.3. Let $E$ be an infinite-dimensional Banach space with a $C^{1}$ strictly convex equivalent norm $\|\cdot\|$ and with a 1-suppression unconditional basis $\left\{e_{n}\right\}_{n \in \mathbb{N}}$, that is a Schauder basis such that

Date: September 2019.

Key words and phrases. Banach space, Morse-Sard theorem, approximation, critical point, diffeomorphic extraction.

The author was supported by Programa Internacional de Doctorado de la Fundación La Caixa-Severo Ochoa 2016 and partially suported by grant MTM2015-65825-P. . 
for every $x=\sum_{j=1}^{\infty} x_{j} e_{j}$ and every $j_{0} \in \mathbb{N}$ we have that

$$
\left\|\sum_{j \in \mathbb{N}, j \neq j_{0}} x_{j} e_{j}\right\| \leq\left\|\sum_{j \in \mathbb{N}} x_{j} e_{j}\right\| .
$$

Let $f: E \rightarrow \mathbb{R}^{d}$ be a $C^{1}$ function and $\varepsilon: E \rightarrow(0, \infty)$ a continuous function. Then for every open set $U$ such that $C_{f} \subset U$ there exists a $C^{1}$ function $\varphi: E \rightarrow \mathbb{R}^{d}$ such that,

(1) $\|f(x)-\varphi(x)\| \leq \varepsilon(x)$ for every $x \in U$.

(2) $f(x)=\varphi(x)$ for all $x \in E \backslash U$.

(3) $D \varphi(x)$ is surjective for all $x \in E$.

The case $c_{0}$ and $l_{p}, 1<p<\infty$ in Theorem 1.1 follow from Theorem 1.2 and Theorem 1.3 respectively. The reader can find the details of why this is so in Remark 4.3 ,

Note that the approximating function that we build does not have any critical point, hence it is an open mapping.

The classical Morse-Sard theorem [18, 22] states that for a given $C^{k}$ function $f: \mathbb{R}^{n} \rightarrow \mathbb{R}^{d}$, if $k \geq$ $\max \{n-d+1,1\}$ then its set of critical values is of Lebesgue measure zero in $\mathbb{R}^{d}$. This set is defined to be the image of the set of critical points, which in turn is defined as $C_{f}=\left\{x \in \mathbb{R}^{n}\right.$ : rank $D f(x)$ is not maximum $\}$.

In general if $E$ and $F$ are Banach spaces, for a differentiable mapping $f: E \longrightarrow F, C_{f}$ stands for the set of points $x \in E$ at which the differential $D f(x)$ is not surjective, and $f\left(C_{f}\right)$ is thus the set of critical values of $f$. In the case that $E$ is of infinite dimension a natural question appears: is it possible to know that $f\left(C_{f}\right)$ is small in any sense by just assuming enough regularity conditions on $f$ ? Unfortunately the answer is no because there exist $C^{\infty}$ smooth functions $f: \ell_{2} \rightarrow \mathbb{R}$ so that their set of critical values $f\left(C_{f}\right)$ contain intervals (see Kupka's counterexample [16]).

A weaker question that we can ask ourselves is if at least any continuous mapping can be uniformly approximated by another one with small critical set of values. Let us mention that for many applications of the Morse-Sard theorem this is sufficient.

The first result of that type was in the case of a continuous function $f: E \rightarrow \mathbb{R}$, where $E$ is a separable Hilbert space. Eells and McAlpin proved in [11] that in such case $f$ can be uniformly approximated by a smooth function $g$ whose set of critical values $g\left(C_{g}\right)$ is of measure zero. This was so called an approximate Morse-Sard result. However in [4], a much stronger result was obtained by Azagra and Cepedello-Boiso: every continuous mapping from $E$, a separable Hilbert space, into $\mathbb{R}^{d}$ can be uniformly approximated by smooth mappings with no critical points. Hájek and Johanis [14] established a similar result for $d=1$ in the case that $E$ is a separable Banach space which contains $c_{0}$ and admits a $C^{k}$ smooth bump function. Also in the case $d=1$, Azagra and Jiménez-Sevilla [7] were able to characterize the class of separable Banach spaces $E$ such that any continuous $f: X \rightarrow \mathbb{R}$ can be uniformly approximated by another one of class $C^{1}$ without any critical point, as those Banach spaces $E$ with separable dual.

Let us comment finally about the very recent paper [3]. In this work, due to Azagra, Dobrowolski and the author, many of the previous results are generalized. It is proved that for the case of $E=c_{0}, \ell_{p}, L^{p}$, $1<p<\infty$, and $F$ a quotient of $E$, any continuous function $f: E \rightarrow F$ can be uniformly approximated by a $C^{k}$ smooth one with no critical points, where $k$ is denoting the order of smoothness of the space $E$ (see [3, Theorems 1.6, 1.7] for more details).

In the present paper we consider a different approach to this problem. Suppose that our given continuous function $f: E \rightarrow \mathbb{R}^{d}$ is already of class $C^{1}$ and we know that its set of critical points $C_{f}$ is 
included in some open set $U$. The question is, are we able not only to uniformly approximate $f$ by another $C^{1}$ function $\varphi$ without critical points but also to make $\varphi$ be equal to $f$ outside $U$ ?

The key will be to use a $C^{1}$-fine approximating result for the function $\left.f\right|_{U}: U \rightarrow \mathbb{R}^{d}$, and this is provided by the results of [19, 5]. This corresponds to Section 3 of the paper.

As a matter of fact, in [19], Moulis was already able to relate $C^{1}$-fine approximations with approximate Morse-Sard type results. She proved that for every $C^{1}$ function $f: E \rightarrow F$, where $E$ is an infinitedimensional separable Hilbert space and $F$ is a separable Hilbert space, and every continuous function $\varepsilon: E \rightarrow(0, \infty)$ there exists a $C^{\infty}$ function $g: E \rightarrow F$ such that $\|f(x)-g(x)\| \leq \varepsilon(x)$, $\| D f(x)-$ $D g(x) \| \leq \varepsilon(x)$ for every $x \in E$ and such that $g\left(C_{g}\right)$ has empty interior in $F$. Obviously we strengthen this conclusion by being able to get $C_{g}=\emptyset$ and considering other Banach spaces, not necessarily Hilbertian. On the other hand for the Hilbert case we cannot write as the target space an infinitedimensional Banach space as Moulis does and also we do not get the approximation in the derivatives.

The proof of both Theorems 1.2 and 1.3 will follow these two steps:

- Step 1: Firstly we construct a $C^{1}$ function $g: U \rightarrow \mathbb{R}^{d}$ such that $\|f(x)-g(x)\| \leq \varepsilon(x) / 2$ and $\|D f(x)-D g(x)\| \leq \varepsilon(x)$ and such that $C_{g}$ either is the empty set for the case of Theorem 1.2 , or is locally contained in a finite union of complemented subspaces of infinite codimension in $E$ for the case of Theorem 1.3 .

- Step 2: We extend the function $g$ to the whole space $E$ by letting it be equal to $f$ outside $U$. Because of the $C^{1}$-fine approximation of Step 1 this extension is still of class $C^{1}$ on $E$. For the case of Theorem 1.2 we are done. For the case of Theorem 1.3 we must find a $C^{1}$ diffeomorphism $h: E \rightarrow E \backslash C_{g}$ which will be the identity outside $U$ and such that $\{\{x, h(x)\}: x \in E\}$ refines $\mathcal{G}$ (in other words, $h$ is limited by $\mathcal{G}$ ), where $\mathcal{G}$ is an open cover of $E$ by open balls $B\left(z, \delta_{z}\right)$ chosen in such a way that if $x, y \in B\left(z, \delta_{z}\right)$ then

$$
\|\varphi(y)-\varphi(x)\| \leq \frac{\varepsilon(z)}{4} \leq \frac{\varepsilon(x)}{2} .
$$

The existence of such a diffeomorphism $h$ follows by a result of Section 2, which is a consequence of some results on extractibility theory from the paper [3, Section 2]. Then, the mapping $\varphi(x):=g(h(x))$ has no critical point, is equal to $f$ outside $U$ and satisfies $\|f(x)-\varphi(x)\| \leq \varepsilon(x)$ for all $x \in E$.

Let us fix now some notations and definitions.

We call $\left\{e_{n}\right\}_{n \in \mathbb{N}}$ the unconditional basis of $E$ and $\left\{e_{n}^{*}\right\}_{n \in \mathbb{N}}$ the associated biorthogonal functionals. Let also $P_{n}: E \rightarrow \operatorname{span}\left\{e_{1}, \ldots, e_{n}\right\}$ be the natural projections defined as $P_{n}\left(\sum_{j=1}^{\infty} x_{j} e_{j}\right)=\sum_{j=1}^{n} x_{j} e_{j}$ and let $K_{u}$ be the unconditional constant for the basis. This constant is defined to be the least number such that for every $\left\{\varepsilon_{j}\right\}_{j=1}^{n} \subset\{-1,+1\}$ and every $\sum_{j=1}^{n} x_{j} e_{j} \in E$,

$$
\left\|\sum_{j=1}^{n} \varepsilon_{j} x_{j} e_{j}\right\| \leq K_{u}\left\|\sum_{j=1}^{n} x_{j} e_{j}\right\| .
$$

Note that $\left\|P_{n}\right\| \leq K_{u}$ for every $n \in \mathbb{N}$. We shall not confuse $K_{u}$ with the suppression unconditional constant $K_{s}$, defined as the least number such that for all (equivalent finite) set $A \subset \mathbb{N},\left\|P_{A}\right\| \leq K_{s}$, where $P_{A}$ represents the projection $P_{A}(x)=\sum_{j \in A} x_{j} e_{j}$. We have the relation $K_{s} \leq K_{u} \leq 2 K_{s}$. Observe also that in the statement of Theorem 1.3 it is required that $K_{s}=1$.

We say that the norm $\|\cdot\|$ locally depends on finitely many coordinates if for every $x \in E$ there exists a natural number $l_{x}$, an open neighbourhood $U_{x}$ of $x$, some functionals $L_{1}, \ldots, L_{l_{x}} \in E^{*}$ and a function $\gamma: \mathbb{R}^{l_{x}} \rightarrow \mathbb{R}$ such that

$$
\|y\|=\gamma\left(L_{1}(y), \ldots, L_{l_{x}}(y)\right)
$$


for every $y \in U_{x}$. In particular we will make use of the fact that if the norm is of class $C^{1}$ and we take $v \in \bigcap_{j=1}^{l_{x}} \operatorname{Ker} L_{j}$, then

for every $y \in U_{x} \backslash\{0\}$.

$$
D\|\cdot\|(y)(v)=\lim _{t \rightarrow 0} \frac{\|y+t v\|-\|y\|}{t}=0
$$

A function $h: E \rightarrow E$ is said to be limited by an open cover $\mathcal{G}$ provided that the set $\{\{x, h(x)\}: x \in E \backslash X\}$ refines $\mathcal{G}$; that is, for every $x \in E \backslash X$, we may find a $G_{x} \in \mathcal{G}$ such that both $x$ and $h(x)$ are in $G_{x}$. When we say that a closed set $X \subset E$ is locally contained in a finite union of complemented subspaces of infinite codimension we mean that for every $x \in X$ there exists an open neighbourhood $U_{x}$ of $x$ and some closed subspaces $E_{1}, \ldots, E_{n_{x}} \subset E$ complemented in $E$ and of infinite codimension such that

$$
X \cap U_{x} \subset \bigcup_{j=1}^{n_{x}} E_{j} .
$$

Finally for a $C^{1}$ function $f: E \rightarrow \mathbb{R}^{d}$, where $f=\left(f^{1}, \ldots f^{d}\right)$, we write its Fréchet derivative at a point $x \in E$ by $D f(x)=\left(D f^{1}(x), \ldots, D f^{d}(x)\right): E \rightarrow \mathbb{R}^{d}$, where each $D f^{i}(x)$ is a continuous linear functional on $E$. If $f$ is $\mathbb{R}$-valued we sometimes simply write $f^{\prime}(x)$ for its derivative.

We will also use indistinctly the symbol $\|\cdot\|$ to denote the norm in $E, E^{*}$ and the euclidean norm in $\mathbb{R}^{d}$.

\section{A COMment about the strong $C^{k}$ extraction property}

In the proof of Theorem 1.3 we will need the following.

Proposition 2.1. Let $E$ be a Banach space with a $C^{k}$ smooth norm. Take an open cover $\mathcal{G}$ of an open set $U$ and a closed set $X \subset U$ that is locally contained in a finite union of complemented subspaces of infinite codimension in $E$. Then there exists a $C^{k}$ diffeomorphism $h: E \rightarrow E \backslash X$ which is the identity outside $U$ and is limited by $\mathcal{G}$.

To achieve this we will use some recent results on diffeomorphic extraction of closed sets that appear in [3, Section 2]. In that paper the next definitions are introduced.

Definition 2.2. A subset $X$ of Banach space $E$ has the strong $C^{k}$ extraction property with respect to an open set $U$ if $X \subseteq U, X$ is relatively closed in $U$, and for every open set $V \subseteq U$, every subset $Y \subseteq X$ relatively closed in $U$ there exists a $C^{k}$ diffeomorphism $\varphi$ from $U \backslash Y$ onto $U \backslash(Y \backslash V)$ which is the identity on $(U \backslash V) \backslash Y$. If in addition for any $\varepsilon>0$ we can ask the diffeomorphism not to move points more than $\varepsilon$ (that is, $\|\varphi(x)-x\| \leq \varepsilon$ for all $x$ ) we will say that $X$ has the $\varepsilon$-strong $C^{k}$ extraction property with respect to $U$.

We will also say that such a closed set $X$ has locally the strong (or $\varepsilon$-strong) $C^{k}$ extraction property if for every point $x \in X$ there exists an open neighbourhood $U_{x}$ of $x$ such that $X \cap U_{x}$ has the strong ( $\varepsilon$-strong respectively) $C^{k}$ extraction property with respect to every open set $U$ for which $X \cap U_{x}$ is a relatively closed subset of $U$.

We have the following properties.

Lemma 2.3. Let us suppose that $X, X_{1}, X_{2} \subset E$ have the $\varepsilon$-strong $C^{k}$ extraction property with respect to an open set $U$ of $E$. Then

(1) For every set $Y \subseteq X$, relatively closed in $U, Y$ has the $\varepsilon$-strong $C^{k}$ extraction property with respect to $U$;

(2) For every open subset $U^{\prime} \subseteq U, X \cap U^{\prime}$ has the $\varepsilon$-strong $C^{k}$ extraction property with respect to $U^{\prime}$.

(3) $X_{1} \cup X_{2}$ has the $\varepsilon$-strong $C^{k}$ extraction property with respect to $U$. 
Proof.

(1) This follows directly from the definition.

(2) See [3, Lemma $2.22(2)]$.

(3) Take $Y \subseteq X_{1} \cup X_{2}$ relatively closed in $U$ and an open set $V \subseteq U$. We want to find a $C^{k}$ diffeomorphism $\varphi$ from $U \backslash Y$ onto $U \backslash(Y \backslash V)$ which is the identity on $(U \backslash V) \backslash Y$ and does not move points more than $\varepsilon$.

Define the sets $Y_{1}=Y \cap X_{1}$ and $Y_{2}=Y \cap X_{2}$, which are relatively closed in $U$ and satisfy $Y_{1} \cup Y_{2}=Y$. In particular by (1) they have the $\varepsilon$-strong $C^{k}$ extraction property with respect to $U$.

(a) There exists a $C^{k}$ diffeomorphism $\varphi_{1}: U \backslash Y_{1} \rightarrow U \backslash\left(Y_{1} \backslash V\right)$ which is the identity on $(U \backslash V) \backslash Y_{1}$ and does not move points more than $\varepsilon / 2$.

(b) For the open set $U \backslash Y_{1}$, using (2) we know that $Y_{2} \cap\left(U \backslash Y_{1}\right)=Y_{2} \backslash Y_{1}$ has the $\varepsilon$-strong $C^{k}$ extraction property with respect to $U \backslash Y_{1}$. Hence there exists a $C^{k}$ diffeomorphism $\varphi_{2}: U \backslash\left(Y_{1} \cup Y_{2}\right) \rightarrow\left(U \backslash Y_{1}\right) \backslash\left(\left(Y_{2} \backslash Y_{1}\right) \backslash V\right)$, which is the identity on $\left(\left(U \backslash Y_{1}\right) \backslash V\right) \backslash\left(Y_{2} \backslash Y_{1}\right)$ and does not move points more than $\varepsilon / 2$.

Observe that

$$
\begin{aligned}
\varphi_{1}\left(\left(U \backslash Y_{1}\right) \backslash\left(\left(Y_{2} \backslash Y_{1}\right) \backslash V\right)\right) & =\left[U \backslash\left(Y_{1} \backslash V\right)\right] \backslash\left[\varphi_{1}\left(\left(Y_{2} \backslash Y_{1}\right) \backslash V\right)\right]= \\
& =\left[U \backslash\left(Y_{1} \backslash V\right)\right] \backslash\left[\left(Y_{2} \backslash Y_{1}\right) \backslash V\right]= \\
& \left.=U \backslash\left(Y_{1} \cup Y_{2}\right) \backslash V\right) .
\end{aligned}
$$

Hence we can define a $C^{k}$ diffeomorphism

$$
\varphi:=\varphi_{1} \circ \varphi_{2}: U \backslash\left(Y_{1} \cup Y_{2}\right) \rightarrow U \backslash\left(\left(Y_{1} \cup Y_{2}\right) \backslash V\right.
$$

which is the identity on $(U \backslash V) \backslash\left(Y_{1} \cup Y_{2}\right)$ and does not move points more than $\varepsilon$.

For this kind of sets the following abstract extractibility result holds.

Theorem 2.4. [3, Theorem 2.24] Let $E$ be a Banach space and $X$ be a closed subset of $E$ which has locally the $\varepsilon$-strong $C^{k}$ extraction property. Let $U$ be an open subset of $E$ and $\mathcal{G}=\left\{G_{r}\right\}_{r \in \Omega}$ be an open cover of $E$. Then there exists a $C^{k}$ diffeomorphism $g$ from $E \backslash(X \backslash U)$ onto $E \backslash X$ which is the identity on $(E \backslash U) \backslash X$ and is limited by $\mathcal{G}$.

Proof of Proposition 2.1. For every $x \in X$ there exists an open neighbourhood $U_{x}$ of $x$ and some closed subspaces $E_{1}, \ldots, E_{n_{x}} \subset E$ complemented in $E$ and of infinite codimension such that

$$
X \cap U_{x} \subseteq \bigcup_{j=1}^{n_{x}} E_{j} .
$$

If $E$ admits an equivalent $C^{k}$ smooth norm it is known (see for instance [3, Theorem 1.4]) that given a complemented subspace $H \subset E$ of infinite codimension and the open set $U_{x}$, then $H \cap U_{x}$ has the $\varepsilon$-strong $C^{k}$ extraction property with respect to any open set $U^{\prime}$ for which $H \cap U_{x}$ is a relatively closed subset of $U^{\prime}$. Therefore thanks to Lemma 2.3 (3) the set $\bigcup_{j=1}^{n_{x}} E_{j} \cap U_{x}$ has the $\varepsilon$-strong $C^{k}$ extraction property with respect to any open set $U^{\prime}$ for which $\bigcup_{j=1}^{n_{x}} E_{j} \cap U_{x}$ is relatively closed on $U^{\prime}$.

Now, using Lemma 2.3 (1), the set $X \cap U_{x} \subseteq \bigcup_{j=1}^{n_{x}} E_{j} \cap U_{x}$ has the $\varepsilon$-strong $C^{k}$ strong extraction property with respect to any open set $U^{\prime}$ for which $X \cap U_{x}$ is relatively closed on $U^{\prime}$. And this means that $X$ has locally the $\varepsilon$-strong $C^{k}$ strong extraction property. To conclude the proof apply Theorem 2.4, noting that we have $X \subset U$ and hence $X \backslash U=\emptyset$. 
For more information about diffeomorphic extraction of closed sets in Banach spaces see for instance [8, 24, 20, 21, 10, 1, 2, 3].

\section{3. $C^{1}$-Fine Approximation CONTROLling the SET of CRitical Points}

Let us proceed with Step 1 of the scheme of the proof of the main Theorems 1.2 and 1.3, described in the introduction. We intend to prove the following two theorems.

Theorem 3.1. Let $E$ be an infinite-dimensional Banach space with an unconditional basis and with a $C^{1}$ equivalent norm that locally depends on finitely many coordinates. Let $U$ be an open subset of $E$, $f: U \rightarrow \mathbb{R}^{d}$ a $C^{1}$ function and $\varepsilon: U \rightarrow(0, \infty)$ a continuous function. Then there exists a $C^{1}$ function $g: U \rightarrow \mathbb{R}^{d}$ such that

(1) $\|f(x)-g(x)\| \leq \varepsilon(x)$ for every $x \in U$.

(2) $\|D f(x)-D g(x)\| \leq \varepsilon(x)$ for every $x \in U$.

(3) $C_{g}=\emptyset$, i.e. $g$ has no critical points.

Theorem 3.2. Let $E$ be an infinite-dimensional Banach space with a $C^{1}$ strictly convex equivalent norm and with a 1-suppression unconditional basis (in particular $K_{u}$-unconditional with $1 \leq K_{u} \leq 2$ ). Let $U$ be an open subset of $E, f: U \rightarrow \mathbb{R}^{d}$ a $C^{1}$ function and $\varepsilon: U \rightarrow(0, \infty)$ a continuous function. Then there exists a $C^{1}$ function $g: U \rightarrow \mathbb{R}^{d}$ such that:

(1) $|f(x)-g(x)| \leq \varepsilon(x)$ for every $x \in U$.

(2) $\|D f(x)-D g(x)\| \leq \varepsilon(x)$ for every $x \in U$.

(3) $C_{g}$ is locally contained in a finite union of complemented subspaces of infinite codimension in E.

The proofs of these results appear in Subsections 3.1 and 3.2 respectively, following the ideas of the papers [19, 5].

However, we must previously introduce an important result that is an easier and slightly different version of [5, Lemma 5]. The proof will mainly be the same but here we want also to study the structure of the critical set of points of the approximating function and we do not care if the approximating function has more regularity than the initial function. If the given function is $C^{1}$, it is enough for the approximating function to be $C^{1}$ as well.

For the readers convenience we present a self-contained proof, even though the arguments are the same as in [19, [5].

Lemma 3.3. Let $E$ and $F$ be a Banach spaces. Suppose that $E$ is infinite-dimensional and has a $K_{u}$-unconditional basis and a $C^{1}$ equivalent norm. Take an open set $U$ of $E$. For every open ball $B_{0}=B\left(z_{0}, r_{0}\right)$ with $B\left(z_{0}, 2 r_{0}\right) \subseteq U$, and for every $C^{1}$ function $f_{1}: U \rightarrow F$ and numbers $\varepsilon, \eta>0$ with $\sup _{x \in B\left(z_{0}, 2 r_{0}\right)}\left\|D f_{1}(x)\right\|<\eta$, there exists a $C^{1}$ function $\Psi: E \rightarrow E$ such that for $f_{2}:=f_{1} \circ \Psi$, we have

(1) $\sup _{x \in B_{0}}\left\|f_{1}(x)-f_{2}(x)\right\|<\varepsilon$.

(2) $\sup _{x \in B_{0}}\left\|D f_{2}(x)\right\|<\left(K_{u}\right)^{2} 8 \eta$.

(3) For every $x \in E$ there exists $n_{0} \in \mathbb{N}$ and a neighbourhood $V_{0}$ of $x$ such that

$$
D \Psi(y)(v)=\sum_{n=1}^{n_{0}}\left[a_{n}(y) D\|\cdot\|\left(y-P_{n-1}(y)\right)\left(v-P_{n-1}(v)\right) y_{n}+\xi_{n}(y) v_{n}\right] e_{n}
$$

for every $v=\sum_{n=1}^{\infty} v_{n} e_{n} \in E$ and $y \in V_{0}$, where $\xi_{n}, a_{n}: V_{0} \rightarrow \mathbb{R}$ are $C^{1}$ functions.

Proof. Choose $0<r<\min \left\{\frac{\varepsilon}{K_{u} \eta}, \frac{r_{0}}{K_{u}}\right\}$. Let $\varphi: \mathbb{R} \rightarrow[0,1]$ be a $C^{\infty}$ smooth function such that $\varphi(t)=1$ if $|t|<\frac{1}{2}, \varphi(t)=0$ if $|t|>1$ and $\varphi^{\prime}(\mathbb{R}) \subseteq[-3,0]$. 
For every $n \in \mathbb{N}$ we define the functions $\xi_{n}: E \rightarrow \mathbb{R}$ and $\Psi: E \rightarrow E$,

$$
\begin{aligned}
\xi_{n}(x) & =1-\varphi\left(\frac{\left\|x-P_{n-1}(x)\right\|}{r}\right), \\
\Psi(x) & =\sum_{n=1}^{\infty} \xi_{n}(x) x_{n} e_{n},
\end{aligned}
$$

where $x=\sum_{n=1}^{\infty} x_{n} e_{n} \in E$. We denote by $P_{0}$ the zero operator.

Fact 3.4. The mapping $\Psi: E \rightarrow \operatorname{span}\left\{e_{n}: n \in \mathbb{N}\right\}$ is well-defined, $C^{1}$ smooth on $E$, and has the following properties:

(1) $\left\|\Psi^{\prime}(x)\right\| \leq\left(K_{u}\right)^{2} 8$ for all $x \in E$;

(2) $\|x-\Psi(x)\| \leq K_{u} r$ for all $x \in E$;

(3) $\Psi\left(B_{0}\right) \subseteq B\left(z_{0}, 2 r_{0}\right)$.

Proof. For any $x \in E$, because $P_{n}(x) \rightarrow x$ and the $\left\|P_{n}\right\|$ are uniformly bounded, there exists a neighbourhood $V_{0}$ of $x$ and an $n_{0} \in \mathbb{N}$ such that $\xi_{n}(y)=0$ for all $y \in V_{0}$ and $n>n_{0}$, and so $\Psi\left(V_{0}\right) \subset \operatorname{span}\left\{e_{1}, \ldots, e_{n_{0}}\right\}$. Thus $\Psi: E \rightarrow \bigcup_{n=1}^{\infty} \operatorname{span}\left\{e_{1}, \ldots, e_{n}\right\}$ is a well-defined $C^{1}$ smooth map. We next compute and estimate its derivative.

We have that

$$
\left(\xi_{n}(y) y_{n}\right)^{\prime}=\xi_{n}^{\prime}(y) y_{n}+\xi_{n}(y) e_{n}^{*}
$$

If $v \in E$ and $y \in V_{0}$

$$
\begin{aligned}
\xi_{n}^{\prime}(y)(v) & =-\varphi^{\prime}\left(\frac{\left\|y-P_{n-1}(y)\right\|}{r}\right) D\|\cdot\|\left(y-P_{n-1}(y)\right)\left(v-P_{n-1}(v)\right) r^{-1}= \\
& =a_{n}(y) D\|\cdot\|\left(y-P_{n-1}(y)\right)\left(v-P_{n-1}(v)\right),
\end{aligned}
$$

where $a_{n}: E \rightarrow \mathbb{R}$ are $C^{1}$ functions, defined by $a_{n}(y)=-\varphi^{\prime}\left(\frac{\left\|y-P_{n-1}(y)\right\|}{r}\right) r^{-1}$. Looking at the expression of $\Psi$ we compute its derivative for every $y \in V_{0}$,

$$
\begin{aligned}
D \Psi(y)(v) & =\sum_{n=1}^{n_{0}}\left[\xi_{n}^{\prime}(y)(v) y_{n}+\xi_{n}(y) v_{n}\right] e_{n}= \\
& =\sum_{n=1}^{n_{0}}\left[a_{n}(y) D\|\cdot\|\left(y-P_{n-1}(y)\right)\left(v-P_{n-1}(v)\right) y_{n}+\xi_{n}(y) v_{n}\right] e_{n}
\end{aligned}
$$

Observe that we have proved (3) of Lemma 3.3 .

Now since $\left|\varphi^{\prime}(t)\right| \leq 3,\left\|\left(I-P_{n-1}\right)^{\prime}(y)\right\| \leq 1+K_{u}$ and the derivative of the norm always has norm one, for all $y$ and all $n$ we get that

$$
\left\|\xi_{n}^{\prime}(y)\right\| \leq\left|\varphi^{\prime}\left(\frac{\left\|y-P_{n-1}(y)\right\|}{r}\right)\right| r^{-1}||\left(I-P_{n-1}\right)^{\prime}(y) \| \leq 3\left(1+K_{u}\right) r^{-1} .
$$


For a fixed $x$, define $n_{1}=n_{1}(x)$ to be the smallest integer with $\left\|x-P_{n_{1}-1}(x)\right\| \leq r$. Then for any $m<n_{1}, \xi_{m}(x)=1$ and $\xi_{m}^{\prime}(x)=0$, and so, for every $v \in B(0,1)$,

$$
\begin{aligned}
\|D \Psi(x)(v)\| & \leq\left\|\sum_{n=n_{1}}^{\infty} \xi_{n}^{\prime}(x)(v) x_{n} e_{n}\right\|+\left\|\sum_{n=1}^{\infty} \xi_{n}(x) v_{n} e_{n}\right\| \leq \\
& \leq K_{u} \sup _{n_{1} \leq n}\left|\xi_{n}^{\prime}(x)(v)\right|\left\|\sum_{n=n_{1}}^{\infty} x_{n} e_{n}\right\|+K_{u} \sup _{n}\left|\xi_{n}(x)\right|\left\|\sum_{n=1}^{\infty} v_{n} e_{n}\right\| \leq \\
& \leq 3 K_{u}\left(1+K_{u}\right) r^{-1}\left\|\sum_{n=n_{1}}^{\infty} x_{n} e_{n}\right\|+K_{u} \leq 4 K_{u}+3\left(K_{u}\right)^{2}<8\left(K_{u}\right)^{2},
\end{aligned}
$$

proving (1).

We next estimate $\|x-\Psi(x)\|$.

$$
\|x-\Psi(x)\|=\left\|\sum_{n \geq n_{1}} x_{n}\left(1-\xi_{n}(x)\right) e_{n}\right\| \leq K_{u} \sup _{n}\left|1-\xi_{n}(x)\right|\left\|\sum_{n \geq n_{1}} x_{n} e_{n}\right\| \leq K_{u} r \leq r_{0},
$$

which proves (2). Lastly, property (3) is immediate from (2) and the choice of $r$.

Going back to the proof of Lemma 3.3 define

$$
f_{2}(x):=f_{1}(\Psi(x)),
$$

which is a $C^{1}$ function. Firstly we have that for every $x \in B_{0}$,

$$
\left\|f_{1}(x)-f_{2}(x)\right\| \leq \eta\|x-\Psi(x)\| \leq \eta K_{u} r<\varepsilon,
$$

using the Lipschitzness of $f_{1}$ in $B\left(z_{0}, 2 r_{0}\right)$.

Secondly for every $x \in B_{0}$,

$$
\left\|D f_{2}(x)\right\| \leq\left\|D f_{1}(\Psi(x))\right\|\|D \Psi(x)\| \leq \eta\left(K_{u}\right)^{2} 8 .
$$

The proof of the Lemma is now complete.

\subsection{Proof of Theorem 3.1,}

Proof of Theorem 3.1. Using the openness of $U$, the continuity of $\varepsilon$ and $f^{\prime}$, the separability of $E$ and the assumption that the norm $\|\cdot\|$ locally depends on finitely many coordinates, we find a covering

$$
\bigcup_{j=1} B\left(x^{j}, r_{j}\right)=U
$$

of $U$ such that

(i) $B\left(x^{j}, 4 r_{j}\right) \subset U$ with $r_{j} \leq 1$ for every $j \in \mathbb{N}$.

(ii) $\varepsilon(x) \geq \frac{\varepsilon\left(x^{j}\right)}{2}$ for every $x \in B\left(x^{j}, 2 r_{j}\right)$.

(iii) $\left\|D f(x)-D f\left(x^{j}\right)\right\| \leq \frac{\varepsilon\left(x^{j}\right)}{\left(K_{u}\right)^{2} 72}$ for every $x \in B\left(x^{j}, 4 r_{j}\right)$.

(iv) For every $j \in \mathbb{N}$ there exist a number $l_{j} \in \mathbb{N}$, some linear functionals $L_{j(1)}, \ldots, L_{j\left(l_{j}\right)}$, and a $C^{1}$ function $\gamma_{j}: \mathbb{R}^{l_{j}} \rightarrow \mathbb{R}$ such that

$$
\|y\|=\gamma_{j}\left(L_{j(1)}(y), \ldots, L_{j\left(l_{j}\right)}(y)\right)
$$

for every $y \in B\left(x^{j}, 2 r_{j}\right)$. 
Now for every $j \in \mathbb{N}$ choose functions $\varphi_{j} \in C^{1}(E ;[0,1])$ with bounded derivative so that $\varphi_{j}(x)=1$ for $x \in B\left(x^{j}, r_{j}\right)$ and $\varphi_{j}(x)=0$ for $x \notin B\left(x^{j}, 2 r_{j}\right)$. We precisely take $\varphi_{j}(x)=\theta_{j}\left(\left\|x-x^{j}\right\|\right)$ where $\theta_{j}: \mathbb{R} \rightarrow[0,1]$ is $C^{\infty}$ and $\theta_{j}^{-1}(1)=\left(-\infty, r_{j}\right]$ and $\theta_{j}^{-1}(0)=\left[2 r_{j}, \infty\right)$. It must be noted here that despite the fact that the norm $\|\cdot\|$ is not differentiable at the origin, the functions $\varphi_{j}$ are $C^{1}$ for every $x \in E$ because in a neighbourhood of $x^{j}$ they are constantly one.

We introduce the following constants,

$$
\begin{aligned}
& \tilde{M}_{k}=\sup _{x \in B\left(x_{k}, 2 r_{k}\right)}\left\|\varphi_{k}^{\prime}(x)\right\|, \\
& M_{j}=\max \left\{1, \sum_{k=1}^{j} \tilde{M}_{k}\right\} .
\end{aligned}
$$

Next define for every $j \in \mathbb{N}$,

$$
h_{j}=\varphi_{j} \prod_{k<j}\left(1-\varphi_{k}\right) .
$$

One can easily check that we have the following properties:

- For every $x \in U$ there exists $n_{x}=\min \left\{m \in \mathbb{N}: x \in B\left(x_{m}, r_{m}\right)\right\}$ such that $1-\varphi_{n_{x}}(x)=0$ and hence $h_{m}(y)=0$ for every $m>n_{x}$ and $y \in B\left(x_{n_{x}}, r_{n_{x}}\right)$.

- $\sum_{j=1}^{\infty} h_{j}(x)=1$ for every $x \in U$.

- $\left\|h_{j}^{\prime}(x)\right\| \leq M_{j}$ for every $j \in \mathbb{N}$ and $x \in B\left(x^{j}, 2 r_{j}\right)$.

In particular $\left\{h_{j}\right\}_{j \in \mathbb{N}}$ is a $C^{1}$ partition of unity which is subordinate to $\left\{B\left(x^{j}, 2 r_{j}\right)\right\}_{j \in \mathbb{N}}$.

For every $j \in \mathbb{N}$ we apply the previous Lemma 3.3 for each ball $B\left(x^{j}, 2 r_{j}\right)$, the function $f_{1}(x)=$ $f\left(x^{j}\right)+D f\left(x^{j}\right)\left(x-x^{j}\right)-f(x)$ and the constants $\frac{\varepsilon\left(x^{j}\right)}{2^{j+3} M_{j}}$ and $\frac{\varepsilon\left(x^{j}\right)}{\left(K_{u}\right)^{2} 72}$ for $\varepsilon$ and $\eta$ respectively. Note that we can apply the Lemma 3.3 because

$$
\sup _{x \in B\left(x^{j}, 4 r_{j}\right)}\left\|D f_{1}(x)\right\|=\sup _{x \in B\left(x^{j}, 4 r_{j}\right)}\left\|D f\left(x^{j}\right)-D f(x)\right\| \leq \frac{\varepsilon\left(x^{j}\right)}{\left(K_{u}\right)^{2} 72} .
$$

The resulting functions from the proof of the lemma will be called $\delta_{j}=f_{1} \circ \Psi_{j}$. In particular we have

$$
\left\|f\left(x^{j}\right)+D f\left(x^{j}\right)\left(x-x^{j}\right)-\delta_{j}(x)-f(x)\right\| \leq \frac{\varepsilon\left(x^{j}\right)}{2^{j+3} M_{j}}
$$

and

$$
\left\|D \delta_{j}(x)\right\| \leq 8 \frac{\varepsilon\left(x^{j}\right)}{72}
$$

for every $x \in B\left(x^{j}, 2 r_{j}\right)$.

Let us define finally

$$
g(x):=\sum_{j=1}^{\infty} h_{j}(x)\left(f\left(x^{j}\right)+D f\left(x^{j}\right)\left(x-x^{j}\right)-\delta_{j}(x)+T_{j}\left(x-x^{j}\right)\right),
$$

where $T_{j}: E \rightarrow \mathbb{R}^{d}$ is a continuous linear surjective operator which we next construct. Define $T_{j}=\left(T_{j}^{1}, \ldots, T_{j}^{d}\right)$ inductively such that for each $i=1, \ldots, d, T_{j}^{i}$ is a non-null element of $E^{*}$ satisfying that

$$
T_{j}^{i} \notin \operatorname{span}\left\{e_{n}^{*}, D f^{k}\left(x^{n}\right), L_{n(1)}, \ldots, L_{n\left(l_{n}\right)}, T_{1}^{k}, \ldots, T_{j-1}^{k}, T_{j}^{1}, \ldots, T_{j}^{i-1}: n \in \mathbb{N}, 1 \leq k \leq d\right\}
$$


(note that it is the span, not the closed span); which can never fill the whole space $E^{*}$ because Banach spaces of infinite dimension can not have a countable Hamel basis. We also impose that their norms are small enough, more precisely,

$$
\left\|T_{j}\right\| \leq \varepsilon\left(x^{j}\right) M_{j}^{-1} 2^{-j-4} \leq \frac{\varepsilon\left(x^{j}\right)}{8} .
$$

An important property that derives from this definition of $T_{j}$ is that the set $\left\{T_{j}^{1}, \ldots, T_{j}^{d}\right\}$ is linearly independent and hence $T_{j}: E \rightarrow \mathbb{R}^{d}$ is a surjective linear operator. We also have that

$$
T_{j}^{i} \notin \operatorname{span}\left\{e_{n}^{*}, D f^{k}\left(x^{n}\right), L_{n(1)}, \ldots, L_{n\left(l_{n}\right)}, T_{1}^{k}, \ldots, T_{j-1}^{k}, T_{j}^{p}: n \in \mathbb{N}, 1 \leq k \leq d, 1 \leq p \leq d, p \neq i\right\} .
$$

Using the expression (3.3) let us check that properties (1), (2) and (3) of the statement of the main theorem are satisfied for this choice of $T_{j}^{i}$.

Firstly if $h_{j}(x) \neq 0$, then $x \in B\left(x^{j}, 2 r_{j}\right)$ and

$$
\begin{aligned}
\| f\left(x^{j}\right)+D f\left(x^{j}\right)\left(x-x^{j}\right)- & \delta_{j}(x)+T_{j}\left(x-x^{j}\right)-f(x) \| \leq \\
& \left.\leq\left\|f\left(x^{j}\right)+D f\left(x^{j}\right)\left(x-x^{j}\right)-\delta_{j}(x)-f(x)\right\|+\left\|T_{j}\left(x-x^{j}\right)\right\|\right) \leq \\
& \leq \frac{\varepsilon\left(x^{j}\right)}{2^{j+3} M_{j}}+\frac{\varepsilon\left(x^{j}\right) 2 r_{j}}{8} \leq \frac{\varepsilon\left(x^{j}\right)}{2} \leq \varepsilon(x) .
\end{aligned}
$$

Therefore for every $x \in U$,

$\|g(x)-f(x)\|=\left\|\sum_{j=1}^{\infty} h_{j}(x)\left(f\left(x^{j}\right)+D f\left(x^{j}\right)\left(x-x^{j}\right)-\delta_{j}(x)+T_{j}\left(x-x^{j}\right)-f(x)\right)\right\| \leq \varepsilon(x) \sum_{j=1}^{n} h_{j}(x)=\varepsilon(x)$.

We have proved (1).

In order to show (2) and 3), let us analyze what the derivative of $g$ looks like, and inspect its critical set.

Claim 3.5. For every $x \in U$ there exist $n, k_{1}, \ldots, k_{n} \in \mathbb{N}$ and a neighbourhood $V_{x}=V \subset B\left(x^{n}, r_{n}\right)$ of $x$ such that:

(i) For every $y \in B\left(x^{n}, r_{n}\right)$,

$D g(y)=\sum_{j=1}^{n} h_{j}^{\prime}(y)\left[f\left(x^{j}\right)+D f\left(x^{j}\right)\left(y-x^{j}\right)-\delta_{j}(y)+T_{j}\left(y-x^{j}\right)\right]+\sum_{j=1}^{n} h_{j}(y)\left[D f\left(x^{j}\right)-D \delta_{j}(y)+T_{j}\right]$.

(ii) For every $y \in V$ and $1 \leq j \leq n, D \delta_{j}(y)(v)=D f\left(\Psi_{j}(y)\right) \circ\left(D \Psi_{j}(y)(v)\right)$ has the form

$$
D f\left(\Psi_{j}(y)\right) \circ\left\{\sum_{n=1}^{k_{j}}\left[a_{n}^{j}(y) D\|\cdot\|\left(y-P_{n-1}(y)\right)\left(v-P_{n-1}(v)\right) y_{n}+\xi_{n}^{j}(y) v_{n}\right] e_{n}\right\} .
$$

Proof. Recall that for every $x \in U$ there is $n_{x}=n=\min \left\{m \in \mathbb{N}: x \in B\left(x_{m}, r_{m}\right)\right\}$ such that $h_{m}(y)=0$ for every $m>n$ and every $y \in B\left(x_{n}, r_{n}\right)$. So expression (3.3) becomes

$$
g(y):=\sum_{j=1}^{n} h_{j}(y)\left(f\left(x^{j}\right)+D f\left(x^{j}\right)\left(y-x^{j}\right)-\delta_{j}(y)+T_{j}\left(y-x^{j}\right)\right)
$$


for all $y \in B\left(x_{n}, r_{n}\right)$. Computing the derivative we get

$D g(y)=\sum_{j=1}^{n} h_{j}^{\prime}(y)\left[f\left(x^{j}\right)+D f\left(x^{j}\right)\left(y-x^{j}\right)-\delta_{j}(y)+T_{j}\left(y-x^{j}\right)\right]+\sum_{j=1}^{n} h_{j}(y)\left[D f\left(x^{j}\right)-D \delta_{j}(y)+T_{j}\right]$,

for every $y \in B\left(x_{n}, r_{n}\right)$.

For every $j=1, \ldots, n$, by (3) of Lemma 3.3, we can find a neighbourhood $V_{x, j} \subset B\left(x_{n}, r_{n}\right)$ of $x$ and a number $k_{j}$ such that such that for every $y \in V_{x, j}$,

$$
\begin{aligned}
& D \delta_{j}(y)(v)=D f\left(\Psi_{j}(y)\right) \circ\left(D \Psi_{j}(y)(v)\right)= \\
& D f\left(\Psi_{j}(y)\right) \circ\left\{\sum_{n=1}^{k_{j}}\left[a_{n}^{j}(y) D\|\cdot\|\left(y-P_{n-1}(y)\right)\left(v-P_{n-1}(v)\right) y_{n}+\xi_{n}^{j}(y) v_{n}\right] e_{n}\right\} .
\end{aligned}
$$

Define then $V_{x}:=\bigcap_{j=1}^{n} V_{x, j} \subset B\left(x_{n}, r_{n}\right)$.

Using equation (3.6) of Claim 3.5, we can write

$$
\begin{aligned}
\|D g(x)-D f(x)\| \leq & \| \sum_{j=1}^{n} h_{j}^{\prime}(x)\left(f\left(x^{j}\right)+D f\left(x^{j}\right)\left(x-x^{j}\right)-\delta_{j}(x)+T_{j}\left(x-x^{j}\right)-f(x) \|+\right. \\
& +\left\|\sum_{j=1}^{n} h_{j}(x)\left(D f\left(x^{j}\right)-D \delta_{j}(x)+T_{j}-D f(x)\right)\right\| \leq \\
\leq & \sum_{j=1}^{n}\left\|h_{j}^{\prime}(x)\right\|\left(\left\|f\left(x^{j}\right)+D f\left(x^{j}\right)\left(x-x^{j}\right)-\delta_{j}(x)-f(x)\right\|+\left\|T_{j}\left(x-x^{j}\right)\right\|\right)+ \\
& +\sum_{j=1}^{n} h_{j}(x)\left(\left\|D f\left(x^{j}\right)-D f(x)\right\|+\left\|D \delta_{j}(x)\right\|+\left\|T_{j}\right\|\right)
\end{aligned}
$$

for every $x \in U$. Let us try to estimate all these quantities. Applying inequality (3.1) and the bound of $\left\|T_{j}\right\|$ given by (3.4) we get

$$
\left\|f\left(x^{j}\right)+D f\left(x^{j}\right)\left(x-x^{j}\right)-\delta_{j}(x)-f(x)\right\|+\left\|T_{j}\left(x-x^{j}\right)\right\| \leq \frac{\varepsilon\left(x^{j}\right)}{2^{j+3} M_{j}}+\frac{\varepsilon\left(x^{j}\right) 2 r_{j}}{2^{j+4} M_{j}}
$$

for every $x \in B\left(x^{j}, 2 r_{j}\right)$. On the other hand $\left\|D f\left(x^{j}\right)-D f(x)\right\| \leq \frac{\varepsilon\left(x^{j}\right)}{\left(K_{u}\right)^{2} 72} \leq \frac{\varepsilon\left(x^{j}\right)}{72}$ by our choice of the partition of unity, and using (3.2) and again (3.4) we have that for every $x \in B\left(x^{j}, 2 r_{j}\right)$,

$$
\left\|D f\left(x^{j}\right)-D f(x)\right\|+\left\|D \delta_{j}(x)\right\|+\left\|T_{j}\right\| \leq \frac{\varepsilon\left(x^{j}\right)}{72}+8 \frac{\varepsilon\left(x^{j}\right)}{72}+\frac{\varepsilon\left(x^{j}\right)}{8}=\frac{\varepsilon\left(x^{j}\right)}{4} .
$$

We also know that the norm of $h_{j}^{\prime}(x)$ is bounded by $M_{j}$ as was indicated when stating the properties of the partition of unity. This fact together with these previous computations allow us to conclude that

$$
\begin{aligned}
\|D g(x)-D f(x)\| & \leq \sum_{j=1}^{n} M_{j}\left(\frac{\varepsilon\left(x^{j}\right)}{2^{j+3} M_{j}}+\frac{\varepsilon\left(x^{j}\right) 2 r_{j}}{2^{j+4} M_{j}}\right)+\sum_{j=1}^{n} h_{j}(x)\left(\frac{\varepsilon\left(x^{j}\right)}{4}\right) \leq \\
& \leq \sum_{j=1}^{n} \frac{\varepsilon\left(x^{j}\right)}{2^{j+2}}+\sum_{j=1}^{n} h_{j}(x) \frac{\varepsilon\left(x^{j}\right)}{4} \leq \frac{\varepsilon(x)}{2}+\frac{\varepsilon(x)}{2}=\varepsilon(x)
\end{aligned}
$$

for every $x \in U$. We have then proved (2) of Theorem 3.1.

Let us focus now on studying the critical set of points of $g$. 
Use Claim 3.5 to choose a vector $x \in U$ for which there exist numbers $n, k_{1}, \ldots, k_{n}$ and a neighbourhood $V=V_{x} \subset B\left(x_{n}, r_{n}\right)$ such that $(i)$ and $(i i)$ of the claim hold. Define also

$$
\tilde{n}:=\max \left\{n, k_{1}, \ldots, k_{n}\right\} .
$$

Take $\left(t_{1}, \ldots, t_{d}\right) \in \mathbb{R}^{d}$ and $y \in V$. Our goal is to find a vector $v \in E$ such that $D g(y)(v)=\left(t_{1}, \ldots, t_{d}\right)$. Once we prove this we will get (3) of Theorem 3.1 .

With $y \in V$ fixed, looking at the formula (3.7) of Claim 3.5, we are interested in the expression of the bounded linear operators $h_{j}^{\prime}(y), D \delta_{j}(y), D f\left(x^{j}\right), T_{j}$ for $j=1, \ldots, n$. Let $m=m_{y}$ be the least number such that $y \in B\left(x^{m}, r_{m}\right)$, that is $h_{m}(y)=1$ (observe that necessarily $m \leq n$ ), then we write equation (3.6) as

$D g(y)=\sum_{j=1}^{m} h_{j}^{\prime}(y)\left[f\left(x^{j}\right)+D f\left(x^{j}\right)\left(y-x^{j}\right)-\delta_{j}(y)+T_{j}\left(y-x^{j}\right)\right]+\sum_{j=1}^{m} h_{j}(y)\left[D f\left(x^{j}\right)-D \delta_{j}(y)+T_{j}\right]$.

We want to find a vector $v \in E$ for which

$$
\left\{\begin{array}{l}
h_{j}^{\prime}(y)(v)=0 \text { for every } 1 \leq j \leq m, \\
D \delta_{j}(y)(v)=(0, \ldots, 0) ; \text { for every } 1 \leq j \leq m, \\
D f\left(x^{j}\right)(v)=\left(D f^{1}\left(x^{j}\right)(v), \ldots, D f^{d}\left(x^{j}\right)(v)\right)=(0, \ldots, 0), \quad \text { for every } 1 \leq j \leq m, \\
T_{j}(v)=(0, \ldots, 0) \text { for every } 1 \leq j<m, \\
h_{m}(y) T_{m}(v)=T_{m}(v)=\left(t_{1}, \ldots, t_{d}\right) .
\end{array}\right.
$$

Let us pay attention to the vectors $y-x^{j}$ and $y-P_{i-1}(y)$, for $1 \leq j \leq m$ and $1 \leq i \leq \tilde{n}$. For simplicity let us rename these vectors as $\left\{z^{1}, \ldots, z^{k_{0}}\right\}$. Each of these elements $z^{k}, 1 \leq k \leq k_{0}$, belongs to some ball $B\left(x^{k^{\prime}}, 2 r_{k^{\prime}}\right)$ (for each $k$ we associate a unique $k^{\prime}$, not necessarily equal to $k$ ). So by using property (iv) from the beginning of the proof there exists a finite number of continuous linear functionals $\left\{L_{k^{\prime}(1)}, \ldots, L_{k^{\prime}\left(l_{k^{\prime}}\right)}\right\}$ and a $C^{1}$ function $\gamma_{k^{\prime}}: \mathbb{R}^{l_{k^{\prime}}} \rightarrow \mathbb{R}$ such that

$$
\left\|z^{k}\right\|=\gamma_{k^{\prime}}\left(L_{k^{\prime}(1)}(y), \ldots, L_{k^{\prime}\left(l_{k^{\prime}}\right)}(y)\right) \text {. }
$$

We intend to take a vector $v \in \bigcap_{j=1}^{l_{k^{\prime}}} \operatorname{Ker} L_{k^{\prime}(j)}$, so that $D\|\cdot\|\left(z^{k}\right)(v)=0$ for every $k=1, \ldots, k_{0}$. For every $i=1, \ldots, d$, let us introduce the finite set of functionals

$$
\begin{aligned}
A_{i}:= & \left\{e_{1}^{*}, \ldots, e_{\tilde{n}}^{*}\right\} \cup\left\{D f^{j}\left(x^{1}\right), \ldots, D f^{j}\left(x^{m}\right): 1 \leq j \leq d\right\} \cup\left\{L_{k^{\prime}(1)}, \ldots, L_{k^{\prime}\left(l_{k^{\prime}}\right)}: 1 \leq k \leq k_{0}\right\} \cup \\
& \cup\left\{T_{1}^{j}, \ldots, T_{m-1}^{j}: 1 \leq j \leq d\right\} \cup\left\{T_{m}^{j}: 1 \leq j \leq d, j \neq i\right\} .
\end{aligned}
$$

By the definition of $T_{m}^{i}$ we have that $T_{m}^{i} \notin \operatorname{span}\left(A_{i}\right)$, which is equivalent to saying that $\bigcap_{a^{*} \in A_{i}}$ Ker $a^{*} \varsubsetneqq$ $\operatorname{Ker} T_{m}^{i}$. Therefore there exists an element $w^{i} \in E$ such that $T_{m}^{i}\left(w^{i}\right) \neq 0$ and $a^{*}\left(w^{i}\right)=0$ for every $a^{*} \in A_{i}$.

For every $i=1, \ldots, d$, take $v^{i}=\frac{t_{i} w^{i}}{T_{m}^{i}\left(w^{i}\right)}$ and define $v:=v^{1}+\cdots+v^{d}$, so we have

$$
T_{m}(v)=\left(T_{m}^{1}(v), \ldots T_{m}^{d}(v)\right)=\left(T_{m}^{1}\left(v^{1}\right), \ldots T_{m}^{d}\left(v^{d}\right)\right)=\left(t_{1}, \ldots, t_{d}\right) .
$$

Moreover, $D\|\cdot\|\left(y-x^{j}\right)(v)=0$ for every $1 \leq j \leq m, D\|\cdot\|\left(y-P_{i-1}(y)\right)(v)=0$ for every $1 \leq i \leq \tilde{n}$, and $D f\left(x^{j}\right)(v)=\left(D f^{1}\left(x^{j}\right)(v), \ldots, D f^{d}\left(x^{j}\right)(v)\right)=(0, \ldots, 0)$ for every $1 \leq j \leq m$. Furthermore, writing $v$ in coordinates, $v=\sum_{j=1}^{\infty} v_{j} e_{j}$ we have that $v_{1}=\cdots=v_{\tilde{n}}=0$.

Recall that $h_{j}(y)=\theta_{j}\left(\left\|y-x^{j}\right\|\right) \prod_{k<j}\left(1-\theta_{k}\left(\| y-x^{k}||\right)\right)$, so

$$
h_{j}^{\prime}(y)(\cdot)=\sum_{k=1}^{j} \gamma_{k, j}(y) D\|\cdot\|\left(y-x^{k}\right)(\cdot)
$$


where $\gamma_{k, j}: E \rightarrow \mathbb{R}$ are $C^{1}$ functions. Hence with our choice of $v$ we have $h_{j}^{\prime}(y)(v)=0$ for every $1 \leq j \leq m$.

On the other hand, looking at formula (3.7) of Claim 3.5, we also get $D \delta_{j}(v)=0$ for every $1 \leq j \leq m$. Finally we also have $T_{j}(v)=\left(T_{j}^{1}, \ldots, T_{j}^{d}(v)\right)=(0 \ldots, 0)$ for every $j<m$, because $T_{j}^{1}, \ldots, T_{j}^{d} \in \bigcap_{i=1}^{d} A_{i}$ for every $j<m$.

Putting all these facts together, we have proved that $D g(y)(v)=\left(t_{1}, \ldots, t_{d}\right)$ and consequently the critical set of points of $g$ is empty.

\subsection{Proof of Theorem $\mathbf{3 . 2}$.}

The essence of the proof will be close to the one of the previous subsection. However there are some important changes. Here we do not rely on a norm that locally depends on finitely many coordinates, but on the property of the basis of being 1-suppression unconditional, which will provide us with the necessary tools to approximate the function $f$ and its derivative $f^{\prime}$ by another function with a small critical set of points.

Proof of Theorem [3.2. $E$ has a separable dual, so it does not contain copies of $l_{1}$ and since it has an unconditional basis, by [17, Theorem 1.c.9] we know that the basis is also shrinking, that is, $\overline{\operatorname{span}}\left\{e_{n}^{*}: n \in \mathbb{N}\right\}=E^{*}$.

Using the openness of $U$, the continuity of $\varepsilon$ and $D f$, and the facts that $\overline{\operatorname{span}}\left\{e_{n}: n \in \mathbb{N}\right\}=E$ and $\overline{\operatorname{span}}\left\{e_{n}^{*}: n \in \mathbb{N}\right\}=E^{*}$, we find a covering

$$
\bigcup_{j=1} B\left(x^{j}, r_{j}\right)=U
$$

of $U$ and continuous linear functionals $F_{j}: E \rightarrow \mathbb{R}^{d}$ for every $j \in \mathbb{N}$ such that:

(i) $B\left(x^{j}, 4 r_{j}\right) \subset U$ with $r_{j} \leq 1$ for every $j \in \mathbb{N}$.

(ii) $\varepsilon(x) \geq \frac{\varepsilon\left(x^{j}\right)}{2}$ for all $x \in B\left(x^{j}, 2 r_{j}\right)$.

(iii) $\left\|D f(x)-D f\left(x^{j}\right)\right\| \leq \frac{\varepsilon\left(x^{j}\right)}{\left(K_{u}\right)^{2} 144}$ for every $x \in B\left(x^{j}, 4 r_{j}\right)$.

(iv) $\left\|F_{j}-D f\left(x^{j}\right)\right\| \leq \frac{\varepsilon\left(x^{j}\right)}{\left(K_{u}\right)^{2} 144}$.

(v) For every $j \in \mathbb{N}$,

$$
\left\{\begin{array}{l}
x^{j}=\sum_{i=1}^{N_{j}} \alpha_{i, j} e_{i}, \\
F_{j}=\left(F_{j}^{1}, \ldots, F_{j}^{d}\right)=\left(\sum_{i=1}^{N_{j}} \beta_{i, j}^{1} e_{i}^{*}, \ldots, \sum_{i=1}^{N_{j}} \beta_{i, j}^{d} e_{i}^{*}\right) .
\end{array}\right.
$$

for some $\alpha_{1, j}, \ldots, \alpha_{N_{j}, j}, \beta_{1, j}^{q}, \ldots, \beta_{N_{j}, j}^{q} \in \mathbb{R}, 1 \leq q \leq d$, where $N_{1} \leq N_{2} \leq \ldots$ is an increasing sequence of natural numbers. Note that we allow some $\alpha_{i, j}$ or $\beta_{i, j}^{q}$ to be null.

At this point we proceed exactly as in the previous subsection, defining the $C^{1}$ partition of unity $\left\{h_{j}\right\}_{j \geq 1}$ subordinate to $\left\{B\left(x^{j}, 2 r_{j}\right)\right\}_{j \geq 1}$, and also the constants $\tilde{M}_{k}$ and $M_{k}$. We also apply Lemma [3.3, exactly in the same way as before, but now to the function $f_{1}(x)=f\left(x^{j}\right)+F_{j}\left(x-x^{j}\right)-f(x)$ and the constants $\frac{\varepsilon\left(x^{j}\right)}{2^{j+3} M_{j}}$ and $\frac{\varepsilon\left(x^{j}\right)}{\left(K_{u}\right)^{2} 72}$ for $\varepsilon$ and $\eta$ respectively, obtaining $\delta_{j}=f \circ \Psi_{j}$.

We define finally

$$
g(x):=\sum_{j=1}^{\infty} h_{j}(x)\left(f\left(x^{j}\right)+F_{j}\left(x-x^{j}\right)-\delta_{j}(x)+T_{j}\left(x-x^{j}\right)\right),
$$

where $T_{j}: E \rightarrow \mathbb{R}^{d}$ is a continuous linear surjective operator that will be defined in the following paragraph. 
Choose a family of pairwise disjoint subsets $\left\{I_{n}\right\}_{n \geq 1}$ of natural numbers such that each $I_{n} \subset \mathbb{N}$ has infinite elements and, if we denote $\mathbb{I}=\bigcup_{n>1} I_{n}$, then $\mathbb{N} \backslash \mathbb{I}$ is infinite. Write also $I_{n}=I_{n}^{1} \cup \cdots \cup I_{n}^{d}$ as a pairwise disjoint union of sets, each of them having again infinite elements. For every $j \in \mathbb{N}$ and $i=1, \ldots, d$ we choose $T_{j}^{i} \in E^{*}$ satisfying that

$$
T_{j}^{i} \in \overline{\operatorname{span}}\left\{e_{n}^{*}: n \in I_{j}^{i}\right\} \backslash \operatorname{span}\left\{e_{n}^{*}: n \in I_{j}^{i}\right\} .
$$

Define $T_{j}:=\left(T_{j}^{1}, \ldots, T_{j}^{d}\right)$ and also assume with no loss of generality that

$$
\left\|T_{j}\right\| \leq \varepsilon\left(x^{j}\right) M_{j}^{-1} 2^{-j-4} \leq \frac{\varepsilon\left(x^{j}\right)}{8} .
$$

Following the computation made for proving Theorem 3.1(1) in the previous subsection, we can check that for every $x \in U$,

$\|g(x)-f(x)\|=\left\|\sum_{j=1}^{\infty} h_{j}(x)\left(f\left(x^{j}\right)-F_{j}\left(x-x^{j}\right)-\delta_{j}(x)+T_{j}\left(x-x^{j}\right)-f(x)\right)\right\| \leq \varepsilon(x) \sum_{j=1}^{n} h_{j}(x)=\varepsilon(x)$,

which proves (1).

To analyze the derivative of $g$ and its set of critical points in order to show (2) and (3) we also have at our disposal the following.

Claim 3.6. For every $x \in U$ there exist $n, k_{1}, \ldots, k_{n} \in \mathbb{N}$ and a neighbourhood $V_{x}=V \subset B\left(x^{n}, r_{n}\right)$ of $x$ such that:

(i) For every $y \in B\left(x^{n}, r_{n}\right)$,

$$
\begin{gathered}
g(y):=\sum_{j=1}^{n} h_{j}(y)\left(f\left(x^{j}\right)+F_{j}\left(y-x^{j}\right)-\delta_{j}(y)+T_{j}\left(y-x^{j}\right)\right), \text { and } \\
D g(y)=\sum_{j=1}^{n} h_{j}^{\prime}(y)\left[f\left(x^{j}\right)+F_{j}\left(y-x^{j}\right)-\delta_{j}(y)+T_{j}\left(y-x^{j}\right)\right]+\sum_{j=1}^{n} h_{j}(y)\left[F_{j}-D \delta_{j}(y)+T_{j}\right] .
\end{gathered}
$$

(ii) For every $y \in V$ and $1 \leq j \leq n, D \delta_{j}(y)(v)=D f\left(\Psi_{j}(y)\right) \circ\left(D \Psi_{j}(y)(v)\right)$ has the form

$$
D f\left(\Psi_{j}(y)\right) \circ\left\{\sum_{n=1}^{k_{j}}\left[a_{n}^{j}(y) D\|\cdot\|\left(y-P_{n-1}(y)\right)\left(v-P_{n-1}(v)\right) y_{n}+\xi_{n}^{j}(y) v_{n}\right] e_{n}\right\} .
$$

Proof. Follow the proof of Claim 3.5.

Using equation (3.10) of Claim 3.6, a straightforward calculation as in the previous subsection gives

$$
\begin{aligned}
\|D g(x)-D f(x)\| \leq & \sum_{j=1}^{n}\left\|h_{j}^{\prime}(x)\right\|\left(\left\|f\left(x^{j}\right)+F_{j}\left(x-x^{j}\right)-\delta_{j}(x)-f(x)\right\|+\left\|T_{j}\left(x-x^{j}\right)\right\|\right)+ \\
& +\sum_{j=1}^{n} h_{j}(x)\left(\left\|F_{j}-D f\left(x^{j}\right)\right\|+\left\|D f\left(x^{j}\right)-D f(x)\right\|+\left\|D \delta_{j}(x)\right\|+\left\|T_{j}\right\|\right) \leq \varepsilon(x)
\end{aligned}
$$

for every $x \in U$. We have thus proved (2) of Theorem 3.2 .

It remains to study the critical set of $g$.

Take a vector $x \in U$. By Claim 3.6 there exist numbers $n, k_{1}, \ldots, k_{n}$ and a neighbourhood $V=V_{x} \subset$ $B\left(x_{n}, r_{n}\right)$ such that (i) and (ii) of the claim hold. Define also

$$
\tilde{n}:=\max \left\{n, N_{n}, k_{1}, \ldots, k_{n}\right\} .
$$


Let us divide the set $\mathbb{N} \backslash \mathbb{I}=\mathbb{J}$ in another disjoint infinite family of subsets $\left\{J_{n}\right\}_{n \geq 1}$, each of them having infinite elements. Consider also the set

$$
A=\left\{y-x^{j}, y-P_{i-1}(y): j=1, \ldots, n i=1 \ldots, \tilde{n}\right\},
$$

and define $k_{0}:=\operatorname{dim}(\{\operatorname{span}(A)\}) \leq n+\tilde{n}$.

In order to establish 3 of Theorem 3.2 our goal is to show that if

$$
y \in V \backslash\left(\bigcup_{k=1}^{k_{0}} \overline{\operatorname{span}}\left\{e_{j}: j=1, \ldots, \tilde{n} \text { or } j \in \mathbb{N} \backslash J_{k}\right\}\right),
$$

and $t=\left(t_{1}, \ldots, t_{d}\right) \in \mathbb{R}$ then there exists a vector $v \in E$ such that $D g(y)(v)=t$. Indeed for every $x \in U$ we would have found a neighbourhood $V_{x}=V$ such that

$$
C_{g} \cap V \subseteq\left(\bigcup_{k=1}^{k_{0}} \overline{\operatorname{span}}\left\{e_{j}: j=1, \ldots, \tilde{n} \text { or } j \in \mathbb{N} \backslash J_{k}\right\}\right)
$$

Fix $y \in V \backslash\left(\bigcup_{k=1}^{k_{0}} \overline{\operatorname{span}}\left\{e_{j}: j=1, \ldots, \tilde{n}\right.\right.$ or $\left.\left.j \in \mathbb{N} \backslash J_{k}\right\}\right)$ and look at the formula of $D g(y)$ given by property (i) of Claim 3.6. We are interested in the expression of the continuous linear operators $h_{j}^{\prime}(y), F_{j}, D \delta_{j}(y), T_{j}$ for $j=1, \ldots, n$. Let $m=m_{y}$ be the least number such that $y \in B\left(x^{m}, r_{m}\right)$, that is $h_{m}(y)=1$ (observe that necessarily $m \leq n$ ), then we may write equation (3.10) as

$$
D g(y)=\sum_{j=1}^{m} h_{j}^{\prime}(y)\left[f\left(x^{j}\right)+F_{j}\left(y-x^{j}\right)-\delta_{j}(y)+T_{j}\left(y-x^{j}\right)\right]+\sum_{j=1}^{m} h_{j}(y)\left[F_{j}-D \delta_{j}(y)+T_{j}\right] .
$$

We need to find a vector $v \in E$ for which

$$
\left\{\begin{array}{l}
h_{j}^{\prime}(y)(v)=0 \text { for every } 1 \leq j \leq m, \\
D \delta_{j}(y)(v)=(0, \ldots, 0) ; \text { for every } 1 \leq j \leq m, \\
F_{j}(v)=\left(F_{j}^{1}(v), \ldots, F_{j}^{d}(v)\right)=(0, \ldots, 0), \text { for every } 1 \leq j \leq m, \\
T_{j}(v)=(0, \ldots, 0) \text { for every } 1 \leq j<m, \\
h_{m}(y) T_{m}(v)=T_{m}(v)=\left(t_{1}, \ldots, t_{d}\right) .
\end{array}\right.
$$

By definition of $y$ there exist $j(1), \ldots, j\left(k_{0}\right)>\tilde{n}$ such that $j(1) \in J_{1}, \ldots, j\left(k_{0}\right) \in J_{k_{0}}$ and $y_{j(1)}, \ldots, y_{j\left(k_{0}\right)} \neq$ 0 . Furthermore the vectors $y-x^{j}$ have their $j(1), \ldots, j\left(k_{0}\right)^{t h}$-coordinates non-null because we had $x^{j} \in$ $\operatorname{span}\left\{e_{1}, \ldots, e_{N_{j}}\right\} \subseteq \operatorname{span}\left\{e_{1}, \ldots, e_{N_{n}}\right\} \subseteq \operatorname{span}\left\{e_{1}, \ldots, e_{\tilde{n}}\right\}$. This implies that the $j(1), \ldots, j\left(k_{0}\right)^{t h}-$ coordinates of all the vectors in the set $A$ (see expression (3.12) ) are non-null.

We will need the following:

Fact 3.7. For every $w=\sum_{j=1}^{\infty} w_{j} e_{j} \in E \backslash\{0\}$ and every $j_{0} \in \mathbb{N}$ we have that

$$
w_{j_{0}} \neq 0 \Longrightarrow D\|\cdot\|(w)\left(e_{j_{0}}\right) \neq 0 \text {. }
$$

Proof. This is a consequence of the facts that the norm is strictly convex and the basis $\left\{e_{n}\right\}_{n \in \mathbb{N}}$ is 1-suppression unconditional. For details see for example [3, Fact 4.5].

Consequently we can assure that

$$
e_{j(k)} \notin \bigcap_{a \in A} \operatorname{Ker}(D\|\cdot\|(a))
$$


for every $1 \leq k \leq k_{0}$. For every $i=1, \ldots, d$, let us define $E_{(m, \tilde{n})}^{i}=\overline{\operatorname{span}}\left\{e_{n}: n>\tilde{n}\right.$ and $\left.n \in \mathbb{J} \cup I_{m}^{i}\right\}$. Since $k_{0}=\operatorname{codim}\left(\bigcap_{a \in A} \operatorname{Ker} D\|\cdot\|(a)\right)$, we can write

$$
E=\left(\bigcap_{a \in A} \operatorname{Ker} D\|\cdot\|(a)\right) \oplus \operatorname{span}\left\{e_{j(1)}, \ldots, e_{j\left(k_{0}\right)}\right\},
$$

so

$$
E_{(m, \tilde{n})}^{i}=\left(\bigcap_{a \in A} \operatorname{Ker} D\|\cdot\|(a) \cap E_{(m, \tilde{n})}^{i}\right) \oplus \operatorname{span}\left\{e_{j(1)}, \ldots, e_{j\left(k_{0}\right)}\right\} .
$$

On the other hand $e_{j(1)}, \ldots, e_{j\left(k_{0}\right)} \in \operatorname{Ker} T_{m}^{i}$ for every $i=1, \ldots, d$. In particular we can find an element

$$
w^{i} \in\left(\bigcap_{a \in A} \operatorname{Ker} D\|\cdot\|(a) \cap E_{(m, \tilde{n})}^{i}\right) \backslash\left(\operatorname{Ker} T_{m}^{i}\right) .
$$

Otherwise we would have $\left(\bigcap_{a \in A} \operatorname{Ker} D\|\cdot\|(a) \cap E_{(m, \tilde{n})}^{i}\right) \subset \operatorname{Ker} T_{m}^{i}$ which implies that $T_{m}^{i}\left(w^{i}\right)=0$ for every $w \in E_{(m, \tilde{n})}^{i}$, a contradiction with the definition of $T_{m}^{i}$.

Let us now mix all these previous ingredients together. The vector $v$ we are looking for is

$$
v:=\sum_{i=1}^{d} \frac{t_{i} w^{i}}{T_{m}^{i}\left(w^{i}\right)}
$$

We obviously have $T_{m}(v)=\left(T_{m}^{1}(v), \ldots, T_{m}^{d}(v)\right)=\left(t_{1}, \ldots, t_{d}\right)$, so it remains to check that $h_{j}^{\prime}(v)=0$, that $D \delta_{j}(v)=F_{j}(v)=(0, \ldots, 0)$ for every $j=1, \ldots, m$ and that $T_{j}(v)=(0, \ldots, 0)$ for every $j<m$. For the $h_{j}^{\prime}$, recall that $h_{j}(y)=\theta_{j}\left(\left\|y-x^{j}\right\|\right) \prod_{k<j}\left(1-\theta_{k}\left(\left\|y-x^{k}\right\|\right)\right)$. So we have that

$$
h_{j}^{\prime}(y)(\cdot)=\sum_{k=1}^{j} \gamma_{k, j}(y) D\|\cdot\|\left(y-x^{k}\right)(\cdot),
$$

where $\gamma_{k, j}: E \rightarrow \mathbb{R}$ are $C^{1}$ functions. The elements $y-x^{j}$ belong to the set $A$ so it is clear that $h_{j}^{\prime}(v)=0$ for every $1 \leq j \leq m$.

For the $D \delta_{j}$, using (3.11) and the facts that the elements $y-P_{i-1}(y)$ belong to the set $A$ and that the coordinates $v_{1}, \ldots, v_{\tilde{n}}=0$, we conclude that $D \delta_{j}(v)=0$ for every $1 \leq j \leq m$.

The fact that $F_{j}(v)=(0, \ldots, 0)$ is clear since

$$
F_{j}=\left(F_{j}^{1}, \ldots, F_{j}^{d}\right)=\left(\sum_{i=1}^{N_{j}} \beta_{i, j}^{1} e_{i}^{*}, \ldots, \sum_{i=1}^{N_{j}} \beta_{i, j}^{d} e_{i}^{*}\right),
$$

$N_{j} \leq N_{n} \leq \tilde{n}$ for every $j=1 \ldots, m$ and $v_{1}, \ldots, v_{\tilde{n}}=0$.

Finally we also have $T_{j}(v)=(0, \ldots, 0)$ for every $j<m$, because $v \in \overline{\operatorname{span}}\left\{e_{n}: n \in \mathbb{J} \cup I_{m}\right\}$ and $\left(\mathbb{J} \cup I_{m}\right) \cap I_{j}=\emptyset$ for every $j<m$.

We have proved that $D g(y)(v)=\left(t_{1} \ldots, t_{d}\right)$ and consequently the critical set of points of $g$ is locally contained in a finite union of complemented subspaces of infinite codimension in $E$.

\section{MAIN RESUlT}

Theorems 3.1 and 3.2 above give us an approximation of a $C^{1}$ function $f: E \rightarrow \mathbb{R}^{d}$ and of its derivative by another function $g: E \rightarrow \mathbb{R}^{d}$ which has a nice critical set of points $C_{g}$. In the case of Theorem 3.1 the term nice means we are in the best situation where $C_{g}=\emptyset$. And in the case of Theorem 3.2 the term nice will mean for us that the closed set $C_{g} \subseteq U$ has the $\varepsilon$-strong $C^{1}$ extraction property with 
respect to $E$, that is, there exists a $C^{1}$ diffeomorphism $h: E \rightarrow E \backslash C_{g}$ such that $h$ is the identity outside $U$ and $h$ refines a given open cover $\mathcal{G}$ of $E$. With these functions at our disposal, and with the help of Proposition 2.1 we can prove our main Theorems 1.2 and 1.3 .

Proofs of Theorems 1.2 and 1.3. Firstly we choose another $C^{1}$ function $\delta: E \rightarrow[0, \infty)$ such that $\delta^{-1}(0)=E \backslash U$ and $\delta(x) \leq \varepsilon(x)$ for every $x \in E$. This is doable because in every separable Banach space with a $C^{1}$ equivalent norm, every closed set is the zero set of a $C^{1}$ function 1 .

By Theorems 3.1 or 3.2 there exists a $C^{1}$ function $g: U \rightarrow \mathbb{R}^{d}$ such that

(1) $\|f(x)-g(x)\| \leq \frac{\delta(x)}{2}$ for every $x \in U$;

(2) $\|D f(x)-D g(x)\| \leq \frac{\delta(x)}{2}$ for every $x \in U$;

(3) $C_{g}=\emptyset$ in the case of Theorem 1.2 , or $C_{g}$ is locally contained in subspaces of infinite codimension in $E$ in the case of Theorem 1.3 .

Let us extend now this function $g: U \rightarrow \mathbb{R}^{d}$ to the whole space $E$ by letting it be equal to $f$ outside $U$. We keep calling this extension by $g$ and it is important to note that this function is still of class $C^{1}$. The only points where this fact could not be clear are those from the boundary of $U$. However the Fréchet derivative of $g$ at those points $x \in \partial U$ exists and is $D f(x)$ because

$$
\begin{aligned}
\limsup _{h \rightarrow 0} \frac{\|g(x+h)-g(x)-D f(x)(h)\|}{\|h\|} \leq & \limsup _{h \rightarrow 0} \frac{\|g(x+h)-f(x+h)+f(x)-g(x)\|}{\|h\|}+ \\
& +\limsup _{h \rightarrow 0} \frac{\|f(x+h)-f(x)-D f(x)(h)\|}{\|h\|}= \\
& =\limsup _{h \rightarrow 0} \frac{\|g(x+h)-f(x+h)\|}{\|h\|}+0 \leq \\
& \leq \lim _{h \rightarrow 0} \frac{\delta(x+h)-\delta(x)}{\|h\|}=0 .
\end{aligned}
$$

Here we are using the facts that $f$ is Fréchet differentiable in $\partial U$ and that $f(x)=g(x)$ and $\delta(x)=$ $\delta^{\prime}(x)=0$ for every $x \in \partial U$.

We have just shown that $g$ is Fréchet differentiable on $E$, but it remains to show that it is $C^{1}$. Straightforwardly for every $x \in \partial U$,

$$
\lim _{y \rightarrow x, y \notin U}\|D g(y)-D f(x)\|=\lim _{y \rightarrow x, y \notin U}\|D f(y)-D f(x)\|=0
$$

and

$$
\limsup _{y \rightarrow x, y \in U}\|D g(y)-D f(x)\| \leq \lim _{y \rightarrow x, y \in U}(\|D g(y)-D f(y)\|+\|D f(y)-D f(x)\|) \leq \lim _{y \rightarrow x, y \in U} \delta(y)=0,
$$

by the continuity of $D f$, property (2) of Theorems 3.1 and 3.2 and because $\delta^{-1}(0)=E \backslash U$.

(1) Case of Theorem 1.2; Define $\varphi=g$ and we obtain that

$$
\|\varphi(x)-f(x)\|,\|D \varphi(x)-D f(x)\| \leq \delta(x) \leq \varepsilon(x)
$$

for all $x \in E$ and $\varphi(x)=f(x)$ for every $x \in E \backslash U$. Besides, it is clear that $\varphi$ does not have any critical point.

\footnotetext{
${ }^{1}$ Wells proved in his thesis 23 that if a separable Banach space $E$ admits a $C^{1}$ smooth Lipschitz bump function, that is a $C^{1}$ non-null function $\lambda: E \rightarrow[0, \infty)$ with bounded derivative and bounded support, then every closed set $X$ of $E$ is the zero set of some $C^{1}$ function. Since a Banach space admitting an equivalent $C^{1}$ norm has a $C^{1}$ smooth Lipschitz bump function our statement is correct.
} 
(2) Case of Theorem 1.3; We will extract the critical set $C_{g}$ in the following way. Observe that $C_{g}$ is a closed set included in $U$ (note that $C_{g} \cap \partial U=\emptyset$ because $D g(x)=D f(x)$ is surjective for every $x \in \partial U$ ), and by (3) of Theorem 3.2 is locally contained in a finite union of complemented subspaces of infinite codimension. Using Proposition 2.1, there exists a $C^{1}$ diffeomorphism $h: E \rightarrow E \backslash C_{g}$ which is the identity outside $U$ and is limited by the open cover $\mathcal{G}$ that we next define. Recall that we have

$$
\|f(x)-g(x)\| \leq \delta(x) / 2
$$

for all $x \in E$. Since $g$ and $\delta$ are continuous, for every $z \in E$ there exists $\eta_{z}>0$ so that if $x, y \in B\left(z, \eta_{z}\right)$ then $\|g(y)-g(x)\| \leq \delta(z) / 4 \leq \delta(x) / 2$. We set $\mathcal{G}=\left\{B\left(x, \eta_{x}\right): x \in E\right\}$.

Finally, let us define

$$
\varphi=g \circ h .
$$

Since $h$ is limited by $\mathcal{G}$ we have that, for any given $x \in E$, there exists $z \in E$ such that $x, h(x) \in B\left(z, \eta_{z}\right)$, and therefore $|g(h(x))-g(x)| \leq \delta(z) / 4$, that is, we have that

$$
\|g(x)-\varphi(x)\| \leq \delta(z) / 4 \leq \delta(x) / 2 .
$$

We obtain that

$$
\|f(x)-\varphi(x)\| \leq \delta(x) \leq \varepsilon(x)
$$

for all $x \in E$. Furthermore $h$ is the identity outside $U$ so $\varphi(x)=g(x)=f(x)$ for every $x \in E \backslash U$. Besides, it is clear that $\varphi$ does not have any critical point: since $h(x) \notin C_{g}$, we have that the linear map $D g(h(x))$ is surjective for every $x \in E$, and $D h(x): E \rightarrow E$ is a linear isomorphism, so $D \varphi(x)=D g(h(x)) \circ D h(x)$ is surjective for every $x \in E$.

The following corollary should be compared with [6, Theorem 1.1], [4, Theorem 1.5] or [7, Corollary 8]. These results are related with the failure of Rolle's theorem in infinite-dimensional Banach spaces.

Corollary 4.1. Let $E$ be a Banach space satisfying the conditions of Theorem 1.2 (in particular $\left.E=c_{0}\right)$. Then for every open set $U$ there exists a $C^{1}$ bump function $\lambda: E \rightarrow[0, \infty)$ whose support is the closure of $U$ and does not have any critical point in $U$.

Remark 4.2. We could have gotten that the approximating function $\varphi$ is of class $C^{k}$ (where $k$ is the order of smoothness of the space $E$ ) inside the open set $U$. To achieve this one should get a version of Lemma 3.3 exactly as in [5, Lemma 5]. Doing this we would get from that lemma that the functions $\delta_{j}(x)$ are of class $C^{k}$. Hence the approximating function $g$ from Theorems 3.1 and 3.2 ,

$$
g(x)=\sum_{j=1}^{\infty} h_{j}(x)\left(f\left(x^{j}\right)+F_{j}\left(x-x^{j}\right)-\delta_{j}(x)+T_{j}\left(x-x^{j}\right)\right)
$$

is a function of class $C^{k}$ on $U$.

Moreover, we can find an extracting diffeomorphism $h: E \rightarrow E \backslash C_{g}$ of class $C^{k}$ by Proposition 2.1, hence $\varphi=g \circ h$ will be a $C^{k}$ mapping on $U$.

\section{Remark 4.3.}

(1) The space $c_{0}$ satisfies the conditions of Theorem 1.2, The supremum norm in $c_{0}$ locally depends on finitely many coordinates, so applying [13, Theorem 1] one gets the existence of an equivalent $C^{\infty}$ smooth norm on $c_{0}$ that locally depends on finitely many coordinates. The space $C(K)$, with $K$ a metrizable countable compactum, also satisfies the conditions of Theorem 1.2 . 
(2) The space $l_{p}$ satisfies the conditions of Theorem 1.3, For every $1<p<\infty$ the canonical norm of $l_{p}$ is

$$
\|x\|=\left\|\sum_{n=1}^{\infty} x_{n} e_{n}\right\|=\left(\sum_{n=1}^{\infty}\left|x_{n}\right|^{p}\right)^{1 / p} .
$$

With this expression it is easy to check that the basis is in fact 1-suppression unconditional with unconditional constant $K_{u}=1$. It is also a norm of class $C^{k}$, where $k$ is defined as follows: $k=\infty$ if $p=2 n, n \in \mathbb{N} ; k=2 n+1$ if $p=2 n+1, n \in \mathbb{N}$, and $k$ is equal to the integer part of $p$ if $p \notin \mathbb{N}$.

\section{ACKnowledgement}

The author wants to thank professor Daniel Azagra for his help and many suggestions in writing this paper.

\section{REFERENCES}

[1] D. Azagra, Diffeomorphisms between spheres and hyperplanes in infinite-dimensional Banach spaces, Studia Math. 125 (1997) no. 2, 179-186.

[2] D. Azagra and T. Dobrowolski, Smooth negligibility of compact sets in infinite-dimensional Banach spaces, with applications, Math. Annalen 312 (1998), no. 3, 445-463.

[3] D. Azagra, T. Dobrowolski and M. García Bravo Smooth approximations without critical points of continuous mappings between Banach spaces, and diffeomorphic extractions of sets, Advances in Mathematics, volume 354, 1 October 2019.

[4] D. Azagra and M. Cepedello, Uniform approximation of continuous mappings by smooth mappings with no critical points on Hilbert manifolds, Duke Math. J. 124 (2004) no. 1, 47-66.

[5] D. Azagra, R. Fry, J. Gómez-Gil, J.A. Jaramillo and M. Lovo, $C^{1}$-fine approximation of functions on Banach spaces with unconditional basis, Quarterly J. Math. 56 (2005) no. 1, 13-20.

[6] D. Azagra, M. Jiménez-Sevilla, The failure of Rolle's theorem in infinite-dimensional Banach spaces, J. Funct. Anal. 182 (2001) 207-226.

[7] D. Azagra and M. Jiménez-Sevilla, Approximation by smooth functions with no critical points on separable infinitedimensional Banach spaces, J. Funct. Anal. 242 (2007), 1-36.

[8] C. Bessaga, Every infinite-dimensional Hilbert space is diffeomorphic with its unit sphere, Bull. Acad. Polon. Sci., Sér. Sci. Math. Astr. et Phys.14 (1966), 27-31.

[9] R. Deville, G. Godefroy, and V. Zizler, Smoothness and renormings in Banach spaces, vol. 64, Pitman Monographs and Surveys in Pure and Applied Mathematics, Longman Scientific \& Technical, Harlow, 1993.

[10] T. Dobrowolski, Smooth and R-analytic negligibility of subsets and extension of homeomorphism in Banach spaces, Studia Math. 65 (1979), 115-139.

[11] J. Eells and J. McAlpin, An approximate Morse-Sard theorem, J. Math. Mech. 17 (1967/1968), $1055-1064$.

[12] M. Fabian, P. Habala, P, Hájek, V. Montesinos, and V. Zizler, Banach space theory. The basis for linear and nonlinear analysis. CMS Books in Mathematics/Ouvrages de Mathématiques de la SMC. Springer, New York, 2011.

[13] P. Hajek, Smooth norms that depend locally on finitely many coordinates, Proc. Amer. Math. Soc. 123 (1995), 3817-3821.

[14] P. Hajek and M. Johanis, Smooth approximations without critical points, Cent. Eur. J. Math. 1 (2003), no. 3, 284-291.

[15] P. Hajek and M. Johanis, Smooth analysis in Banach spaces, De Gruyter Series in Nonlinear Analysis and Applications, Berlin/Boston 2014.

[16] I. Kupka, Counterexample to the Morse-Sard theorem in the case of infinite-dimensional manifolds, Proc. Amer. Math. Soc. 16 (1965), 954-957.

[17] J. Lindenstrauss, and L. Tzafriri, Classical Banach spaces. I. Sequence spaces. Ergebnisse der Mathematik und ihrer Grenzgebiete, Vol. 92. Springer-Verlag, Berlin-New York, 1977.

[18] A. Morse, The behavior of a function on its critical set, Annals of Math. 40 (1939), 62-70.

[19] N. Moulis, Approximation de fonctions différentiables sur certains espaces de Banach, Ann. Inst. Fourier, Grenoble 21 (1971), 293-345.

[20] P.L. Renz, Smooth extensions and extractions in infinite dimensional Banach spaces. Thesis (Ph.D.)-University of Washington, 1969.

[21] P. Renz, Smooth extensions in infinite dimensional Banach spaces, Trans. Amer. Math. Soc. 168 (1972), $121-132$. 
[22] A. Sard, The measure of the critical values of differentiable maps, Bull. Amer. Math. Soc. 48 (1942), 883-890.

[23] J.C. Wells, Smooth Banach spaces and approximations, Thesis (Ph.D.) California Institute of Technology, 1969.

[24] J.E. West, The diffeomorphic excision of closed local compacta from infinite-dimensional Hilbert manifolds, Compositio Math. 211969 271-291.

ICMAT (CSIC-UAM-UC3-UCM), CAlle Nicolás Cabrera 13-15. 28049 Madrid SPAIN

E-mail address: miguel.garcia@icmat.es 